\title{
Coexpression network analysis reveals an MYB transcriptional activator involved in capsaicinoid biosynthesis in hot peppers
}

\author{
Binmei Sun ${ }^{1}$, Xin Zhou ${ }^{1,2}$, Changming Chen', Chengjie Chen', Kunhao Chen ${ }^{3,4}$, Muxi Chen ${ }^{3,4}$, Shaoqun Liu', \\ Guoju Chen ${ }^{1}$, Bihao Cao ${ }^{1}$, Fanrong Cao ${ }^{1}$, Jianjun Lei ${ }^{1,5}$ and Zhangsheng Zhu ${ }^{1,6}$
}

\begin{abstract}
Plant biosynthesis involves numerous specialized metabolites with diverse chemical natures and biological activities. The biosynthesis of metabolites often exclusively occurs in response to tissue-specific combinatorial developmental cues that are controlled at the transcriptional level. Capsaicinoids are a group of specialized metabolites that confer a pungent flavor to pepper fruits. Capsaicinoid biosynthesis occurs in the fruit placenta and combines its developmental cues. Although the capsaicinoid biosynthetic pathway has been largely characterized, the regulatory mechanisms that control capsaicinoid metabolism have not been fully elucidated. In this study, we combined fruit placenta transcriptome data with weighted gene coexpression network analysis (WGCNA) to generate coexpression networks. A capsaicinoid-related gene module was identified in which the MYB transcription factor CaMYB48 plays a critical role in regulating capsaicinoid in pepper. Capsaicinoid biosynthetic gene (CBG) and CaMYB48 expression primarily occurs in the placenta and is consistent with capsaicinoid biosynthesis. CaMYB48 encodes a nucleus-localized protein that primarily functions as a transcriptional activator through its C-terminal activation motif. CaMYB48 regulates capsaicinoid biosynthesis by directly regulating the expression of CBGs, including AT3a and Kas/a. Taken together, the results of this study indicate ways to generate robust networks optimized for the mining of CBG-related regulators, establishing a foundation for future research elucidating capsaicinoid regulation.
\end{abstract}

\section{Introduction}

Hot pepper (Capsicum spp.) is the most popular vegetable and ingredient in the world because of its attractive spicy flavor ${ }^{1,2}$. The pungent taste originates from a group of alkaloid compounds, capsaicinoids, which are uniquely produced by the Capsicum genus ${ }^{2}$. To date, more than 22 capsaicinoids have been identified in hot pepper; capsaicinoid (Cap) and dihydrocapsaicin (DhCap) are the two major components and account for $\sim 90 \%$ of the total capsaicinoid content $^{3-5}$. Capsaicinoids are exclusively

Correspondence: Jianjun Lei (jjlei@scau.edu.cn) or

Zhangsheng Zhu (zhuzhangsheng0@163.com)

${ }^{1}$ Key Laboratory of Biology and Genetic Improvement of Horticultural Crops (South China), Ministry of Agriculture and Rural Affairs, College of Horticulture, South China Agricultural University, Guangzhou 510642, China

${ }^{2}$ Jiangxi Agricultural Engineering College, Zhangshu 331200 Jiangxi, China

Full list of author information is available at the end of the article synthesized in the pepper placenta, mainly from 16 days post-anthesis (DPA) to the mature green stage through the condensation reaction of the precursors derived from phenylpropanoid and the branched-chain fatty acid pathway ${ }^{6-8}$. Capsaicinoid biosynthetic genes (CBGs), such as CCoAMT, AMT, BCAT, KasIa, ACL, KR, FatA, and $A T 3 a$, have been identified as being involved in capsaicinoid biosynthesis ${ }^{3,8-13}$. In nature, pepper synthesizes capsaicinoids for antifungal and antibacterial activities, and these compounds also act as a deterrent to mammals ${ }^{14}$. For humans, capsaicinoids function as bioactive compounds because of their many benefits: they are not only effective in treating many diseases, such as cancer and obesity, as well as pain ${ }^{15,16}$, but are also added to food for sterilization ${ }^{17}$ and widely applied in riot control and personal defense spray agents ${ }^{18}$. Since capsaicinoids have 
considerable application value and commercial purposes, several methods have been employed to increase the capsaicinoid content ${ }^{5,19,20}$. However, the capsaicinoid biosynthesis process is strictly switched spatially and temporally, and the expression of CBGs in pepper is precisely regulated at the transcriptional level ${ }^{4,21}$. Altering the expression of key CBGs in pepper seems to be effective in improving capsaicinoid contents. In highly pungent peppers, the transcriptional level of CBGs (e.g., $A M T$, KasIa, and $A T 3 a$ ) is consistently higher than that in weakly pungent cultivars ${ }^{3,5,21}$. Remarkably, the expression level of a major transcription factor usually affects the transcript level of all genes in the biosynthetic pathway, which will ultimately change the accumulation of compounds $^{5,22,23}$. For example, Catharanthus roseus ORCA3 is a crucial transcription factor that regulates the expression of terpenoid indole alkaloid genes, and overexpression of ORCA3 results in 3.2-fold-increased accumulation of terpenoid indole alkaloids ${ }^{24}$. Similarly, in horticultural plants, such as tomato (Solanum lycopersi$(\mathrm{cum})^{25}$, tea (Camellia sinensis) ${ }^{26}$, and orange (Citrus sinensis $)^{27}$, high accumulation of health-promoting anthocyanins, which arise from upregulation of the anthocyanin-related MYB transcriptional activator, leads to anthocyanin biosynthetic gene expression simultaneously. Although the Solanaceae-specific MYB transcription factor MYB31 was determined to be involved in capsaicinoid biosynthesis ${ }^{5,28}$, the transcriptional regulation of capsaicinoid biosynthesis has not been fully elucidated. Therefore, the identification of capsaicinoid biosynthesis-related transcription factors is needed.

A number of research efforts have focused on the biosynthesis of capsaicinoids to facilitate manipulations of pepper capsaicinoid content. Previously, the identification of genes relevant to capsaicinoid biosynthesis was performed via a combination of genetic fine mapping and reverse genetic and biochemistry approaches ${ }^{3,8}$. However, this method was hindered by the construction of a mapping population, which is laborious, and genetic transformation is highly difficult. In subsequent research, considerable progress has been achieved in RNA sequencing (RNA-seq). Identification of capsaicinoid biosynthesis candidate genes through transcriptomic data appears to be a more efficient strategy ${ }^{4,11}$. Gene coexpression network analysis (GCNA) is a systems biology approach for describing the correlation patterns between genes across large-scale gene expression profiling data ${ }^{29}$. To date, GCNA has been successfully applied in various biological contexts. In particular, these studies have provided key insights into plant secondary metabolism processes $^{30}$. For instance, GCNA was used as a valid approach to identify biosynthetic genes from mayapple that complete the biosynthetic pathway to the etoposide aglycone $^{31}$. In parallel, this technique also highlighted the role of GAME9 in regulating steroidal alkaloid biosynthesis in tomato and potato ${ }^{32}$. As one of the GCNA-based approaches, weighted GCNA (WGCNA) is a correlationbased technique that describes and visualizes coexpression networks between genes using transcriptomic data ${ }^{33}$. Currently, WGCNA is the most popular method used to identify and dissect gene modules in specific biological processes in a variety of plant species ${ }^{34}$. Therefore, utilization of WGCNA will facilitate the identification of novel potential regulators related to capsaicinoid biosynthesis. Despite these enlightening reports, little is known about the use of WGCNA to identify transcription factors involved in the regulation of capsaicinoid metabolism.

Capsaicinoid biosynthesis exclusively occurs in the pepper fruit placenta and in response to combinatorial developmental cues that are controlled at the transcriptional level. The availability of a large amount of RNA-seq data in pepper provides an ideal approach for investigating the regulation of capsaicinoid biosynthesis. In this study, through WGCNA, we identified a module related to capsaicinoid. In this module, we identified and characterized an R2R3 MYB transcription factor, CaMYB48, involved in capsaicinoid biosynthesis. CaMYB48 positively regulates capsaicinoid accumulation in pepper by directly activating CBGs. This study describes ways to generate robust networks optimized for the identification of CBG-related regulators, establishing a foundation for further research investigating the regulatory network of capsaicinoids.

\section{Results}

Identification of the capsaicinoid-related module

Capsaicinoid biosynthesis primarily occurs in the pepper fruit placenta. Capsaicinoid levels are highly dynamic during fruit development and appear to be influenced by the ontogenetic trajectory of the fruit ${ }^{4,5}$. Capsaicinoids begin to accumulate at the fruit developmental stage of 16 DPA and peak at the mature stage ( 40 DPA). Accordingly, key CBGs (i.e., $A M T$, KasIa, and $A T 3 a$ ) are also highly expressed in the placenta from 16 DPA to the mature green stage (approximately 30 DPA) during pepper placenta development. This highly dynamic process is precisely governed at the transcriptional level. To understand the landscape of transcription regulation of capsaicinoid biosynthesis, transcriptomic data of the pungent cultivar CM334 (Capsicum annuum) and nonpungent cultivar ECW (C. annuum) placenta at seven different developmental stages were retrieved from previous studies ${ }^{4}$. Coexpression networks were generated via the WGCNA package, and the combinational 14placenta-development-stage network incorporated 26 clusters of coexpressed genes (Fig. 1a and Supplementary Fig. 1). The eigengenes were recognized as the first principal component of a cluster and can be thought of as 


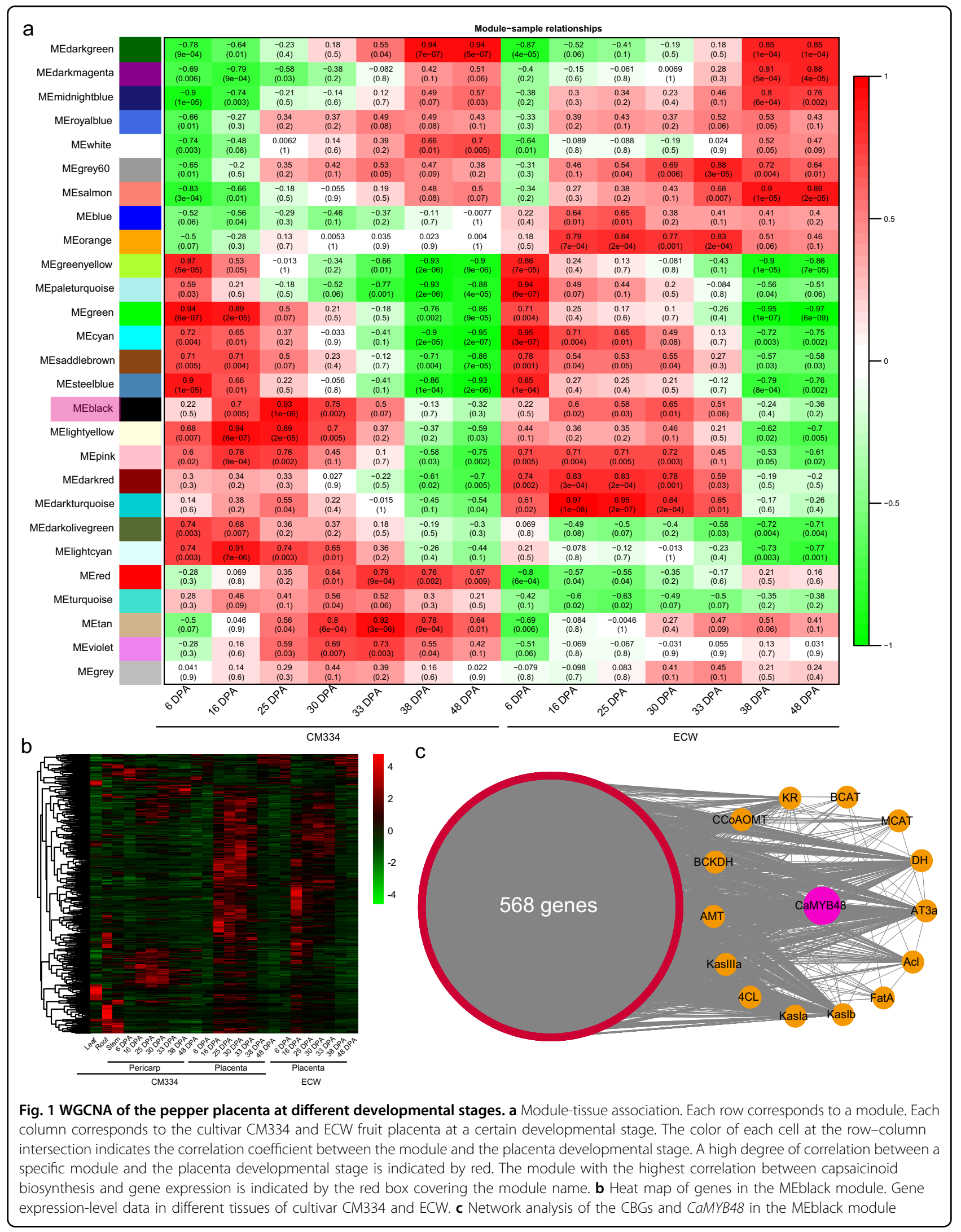


representative of a cluster's expression profile. The expression level of each cluster's eigengene in each of the placenta tissues is plotted in a heat map (Fig. 1a), which enables easy visualization of the cluster placenta developmental stage association. Capsaicinoid biosynthesis occurs from 16 DAP to 30 DPA (mature green stage), and many CBGs are also highly expressed during this stage. Among the 26 distinct modules, the MEblack module had an expression pattern tightly correlated with capsaicinoid biosynthesis (Fig. 1b and Supplementary Figs. 2, 3). We found that in both CM334 and ECW, the genes in cluster MEblack were expressed most highly from 16 DPA to 30 DPA. In addition, the MEblack genes of different tissues were plotted in a heat map, and most of these genes exhibited a placenta-preferred or placenta-specific pattern (Fig. 1b). In total, 568 genes were included in this module: 14 CBGs, including key CBGs, such as AMT, KasIa, and $A T 3 a$ (Fig. 1c and Supplementary Table 1). In addition, 20 out of 568 genes were identified as transcription factors (Supplementary Table 2). Notably, among these transcription factors was an MYB transcription factor, CA11g12490, referred to in this study as CaMYB48, which was tightly associated with the MEblack module (kME $P$ value $=1.42 \mathrm{E}-08$ ). Moreover, CaMYB48 was found to be highly coexpressed with CBGs. Since a vast number of MYB transcription factors have been characterized as being involved in regulating plant specialized metabolite biosynthesis $^{35}$, we speculate that CaMYB48 might play an important role in regulating capsaicinoid biosynthesis.

\section{CaMYB48 and CBG expression patterns are spatiotemporally specific}

To better understand the relationship between CaMYB48 and capsaicinoid biosynthesis pathway gene expression (Fig. 2a), different sets of raw pepper transcriptome data were retrieved from previous studies ${ }^{4,36}$. The results indicated that the expression of CBGs in pungent cultivar CM334 was gene-dependent (Fig. 2b). The upstream biosynthetic pathway genes (e.g., $P A L$ and C4H) did not display tissue-specific expression patterns. In contrast, most downstream CBGs exhibited a spatiotemporally specific expression pattern, i.e., high expression in the 16-30 DPA placenta. Notably, a high expression level of CaMYB48 was also detected at 16-30 DPA in the placenta (Fig. 2b). However, a considerable expression level of CaMYB48 was also detected in the pericarp during this stage, indicating that CaMYB48 may also have certain functions in the pericarp. To further reveal whether CaMYB48 expression and CBG expression exhibit similar patterns among different cultivars, transcriptome data of the 6421 pungent inbred line at different fruit developmental stages (11 stages) were also retrieved from previous studies ${ }^{36}$. Consistent with the results observed in CM334, CaMYB48 was highly coexpressed with downstream CBGs. Taken together, these results suggested that CaMYB48 might regulate the coexpressed CBGs to regulate capsaicinoid biosynthesis.

\section{CaMYB48 is associated with capsaicinoid biosynthesis}

To characterize the capsaicinoid contents in the fruit placenta of elite inbred line 59 (C. аnnиum) during different developmental stages, we adopted highperformance liquid chromatography (HPLC) to analyze extracts from six developmental stages $(10,16,25,33,38$, and 45 DPA) (Fig. 3a). The abundances of two major capsaicinoids, Cap and DhCap, gradually increased during development, with a rapid increase from 16 DPA to 33 DPA and a peak at 38 DPA (Fig. 3b). Real-time quantitative reverse transcription PCR (qRT-PCR) analysis was performed to dissect the relationship between CaMYB48 and capsaicinoid biosynthesis. The expression pattern of CaMYB48 and CBGs was displayed in a tissue- and development-dependent manner. The results revealed a high abundance of CaMYB48 detected in the placenta from 16 DPA to 33 DPA, and the key CBGs AMT, KasIa, and $A T 3 a$ were also highly expressed at these stages (Fig. 3c), implying that CaMYB48 might govern the expression of these genes. However, we found that CaMYB48 also has considerable expression levels in other tissues, such as roots, pericarps, and flowers, indicating that it functions in these tissues. Taken together, these results strongly indicate that CaMYB48 is a candidate regulator of capsaicinoid biosynthesis.

\section{CaMYB48 encodes a nucleus-localized R2R3 MYB transcription factor}

The full-length coding sequence of CaMYB48 was cloned from the placenta of the 59 inbred line. Nucleotide sequence analysis indicated that the coding sequence of CaMYB48 was 669 bp in length, and the deduced amino acid sequence was 222 amino acids (Fig. 4a). The MYB transcription factors that shared the highest sequence identity with CaMYB48 were retrieved from Solanum lycopersicum, Solanum tuberosum, Nicotiana tabacum, Artemisia annua, and Arabidopsis thaliana. Sequence analysis indicated that the protein contained R2 and R3 MYB domains within the N-terminus (Fig. 4a), which play an important role in DNA binding. To dissect the potential function of CaMYB48, phylogenetic analysis was performed. The MYB transcription factors that shared the highest sequence identity with CaMYB48 from other plant species were retrieved from public databases, while some functionally identified MYB transcription factors were also adopted for analysis. As shown in the phylogenetic tree, within the CaMYB48 clade, the nearest MYB transcription factors from Solanaceae plants, i.e., SlMYB48 (Solanum lycopersicum), StMYB48 (Solanum tuberosum), and NtMYB48 (Nicotiana tabacum), shared a 


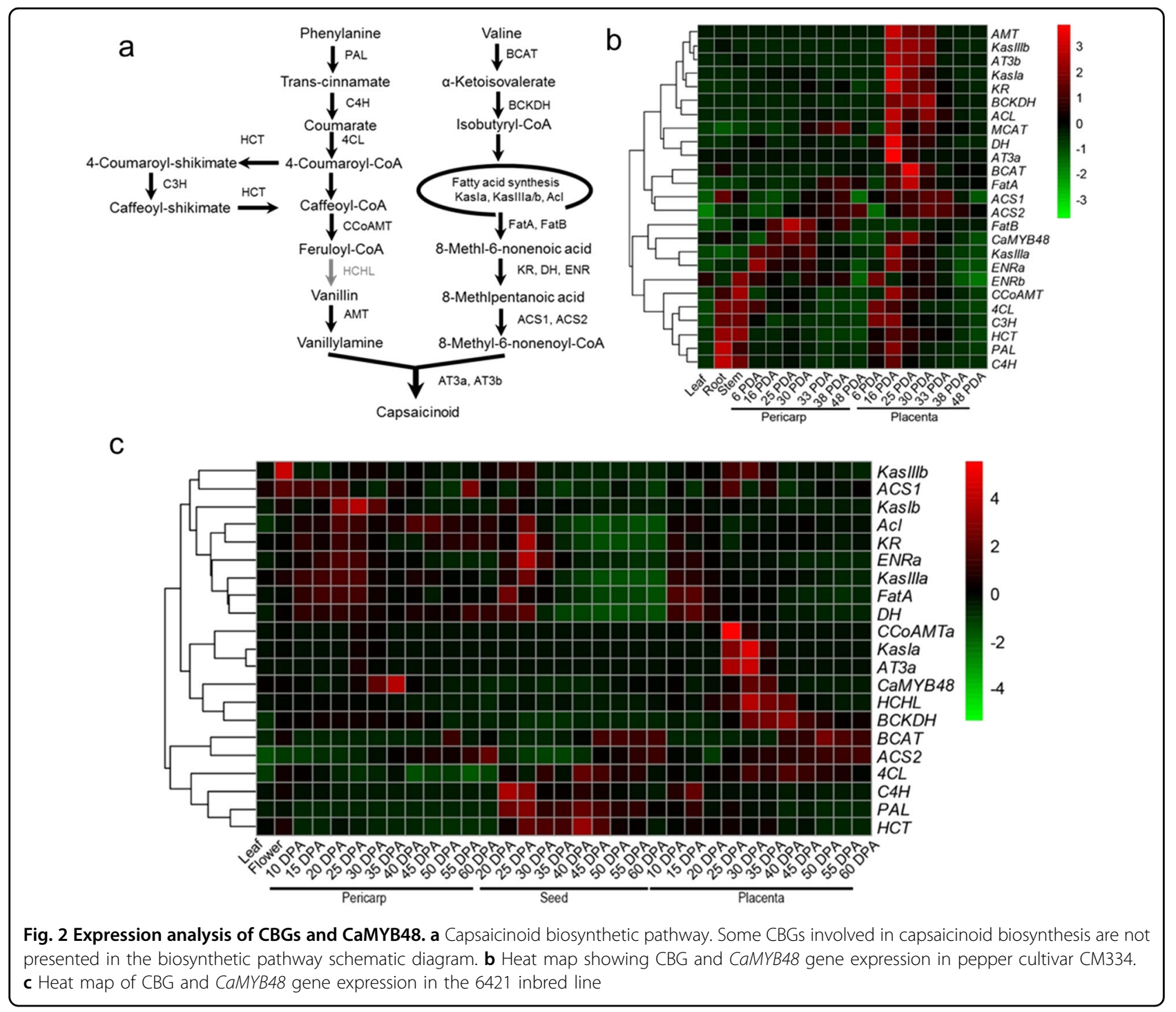

sequence identity of more than 90\% (Fig. 4b). However, the MYB functions in these Solanaceae plants are unknown. The most closely related MYB transcription factor from Arabidopsis thaliana was AtMYB48, which showed only a $53 \%$ amino acid sequence identity, and its function was not characterized.

Transcription factors execute transcriptional regulation depending on localization in the nucleus. To investigate the subcellular localization of CaMYB48, the coding sequence of CaMYB48 was fused in frame with the GFP gene to generate a 35S:CaMYB48-GFP construct (Fig. 4c). The SV40 nucleus-localized signal (NLS) fused with the DsRed protein to generate the nuclear marker. 35S:GFP or 35S:CaMYB48-GFP was coexpressed with DsRed in Nicotiana benthamiana leaf epidermal cells. Using confocal microscopy, we observed that the CaMYB48-GFP fusion protein colocalized with DsRed (Fig. 4c), confirming that CaMYB48 functions as a nucleus-localized transcription regulator. In contrast, in the empty vector, the GFP signal was observed in both the cytoplasm and nucleus of the epidermal cell.

\section{CaMYB48 functions as a transcriptional activator}

To identify the potential conserved motif in CaMYB48, the amino acid sequence was searched via the Multiple Em for Motif Elicitation (MEME) database. In addition to the $\mathrm{R} 2$ and $\mathrm{R} 3 \mathrm{MYB}$ repeats in the $\mathrm{N}$-terminus, an acidic domain was also observed in the C-terminus (Fig. 5a). To determine how these motifs associate with CaMYB48 transactivation ability, we performed a transactivation activity assay in yeast and plants to test the transcriptional activation activity of CaMYB48. Based on the presence of conserved domains, full-length or truncated CaMYB48 was fused in frame with the GAL4 DNA-binding domain $(\mathrm{BD})$ in the pGBKT7 (BD) vector, and the constructs were transformed into the $\mathrm{Y} 2 \mathrm{H}$ Gold yeast strain. The growth 


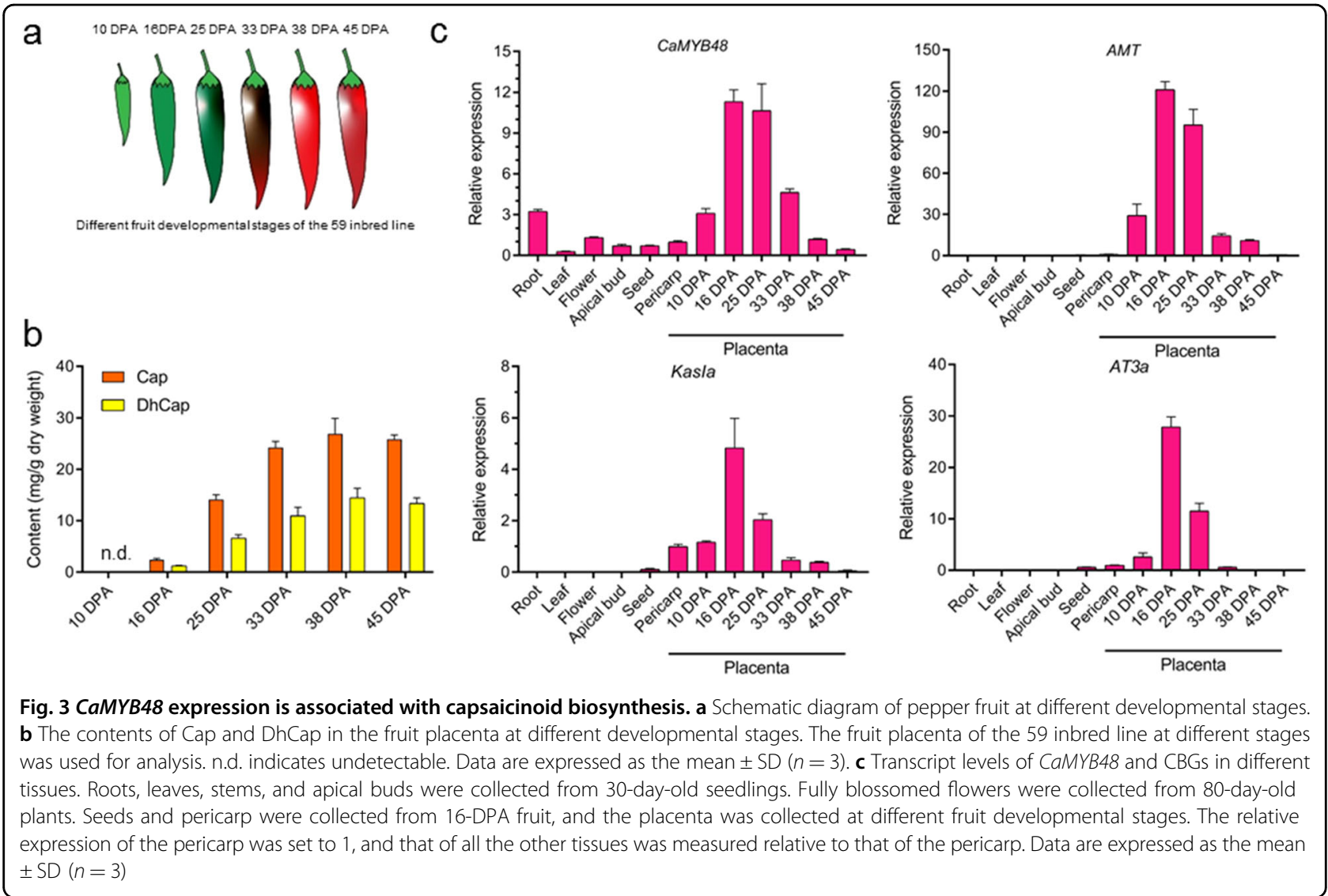

of yeast carrying BDCaMYB48 on SD/-Trp/-His/-Ade selective medium along with an $\mathrm{x}-\alpha$-gal assay indicated that the CaMYB48 protein has strong activation activity (Fig. 5b). To determine how the structure of CaMYB48 influences its activation activity, the CaMYB48 protein was divided into two major parts based on conserved domain analysis: the R2R3 repeat domain (1-115 aa) and the C-terminal domain (116-222 aa). Analyzing truncated CaMYB48 in the yeast assay indicated that aa 168-191 were essential for the activation activity of CaMYB48 (Fig. $5 b)$. To explore whether this transcriptional activation ability is mediated in plants, a dual-luciferase (LUC) reporter assay was performed. The full-length or truncated CaMYB48 was fused in frame with the GAL4 BD to generate an effector (Fig. 5c). The effector and reporter were coexpressed in Nicotiana benthamiana leaves via Agrobacterium-mediated transformation. Compared with the empty control, CaMYB48 strongly enhanced the relative activity of firefly LUC, suggesting that CaMYB48 has transcriptional activity in $N$. benthamiana (Fig. 5c). Further analysis indicated that the C-terminal acidic amino acid region was responsible for executing the transcriptional activity of CaMYB48 (Fig. 5c). Taken together, these data indicate that CaMYB48 functions as a transcriptional activator and that the $\mathrm{C}$-terminal acidic amino acid region is necessary and sufficient for its transcriptional activity in yeast and $N$. benthamiana.

\section{Downregulation of CaMYB48 decreases CBG expression and capsaicinoid content}

To reveal the function of CaMYB48 in peppers, a lossof-function analysis of CaMYB48 via virus-induced gene silencing (VIGS) was carried out to silence CaMYB48 in the 59 inbred line. The silencing efficiency was evaluated by qRT-PCR analysis of the CaMYB48 transcription level in the placenta at the developmental stage of 16 DPA. Compared with that in the control, the CaMYB48 transcription level in the CaMYB48-silenced plants was reduced threefold (Fig. 6a). The expression of the upstream CBG (e.g., $P A L$ and $C 4 H$ ), which is derived from the phenylpropanoid pathway, changed slightly in both the control and CaMYB48-silenced plants. In contrast, the expression of the downstream CBG changed significantly, and the percentage of genes that decreased transcript levels ranged from $\sim 60$ to $90 \%$ (Fig. 6a). Consistent with the downregulation of CaMYB48 and CBGs in CaMYB48-silenced plants, the Cap and DhCap contents decreased by $43 \%$ and $45 \%$, respectively, compared to those in the control plants (Fig. 6b). Most of the downregulated CBGs were consistent with the coexpression 


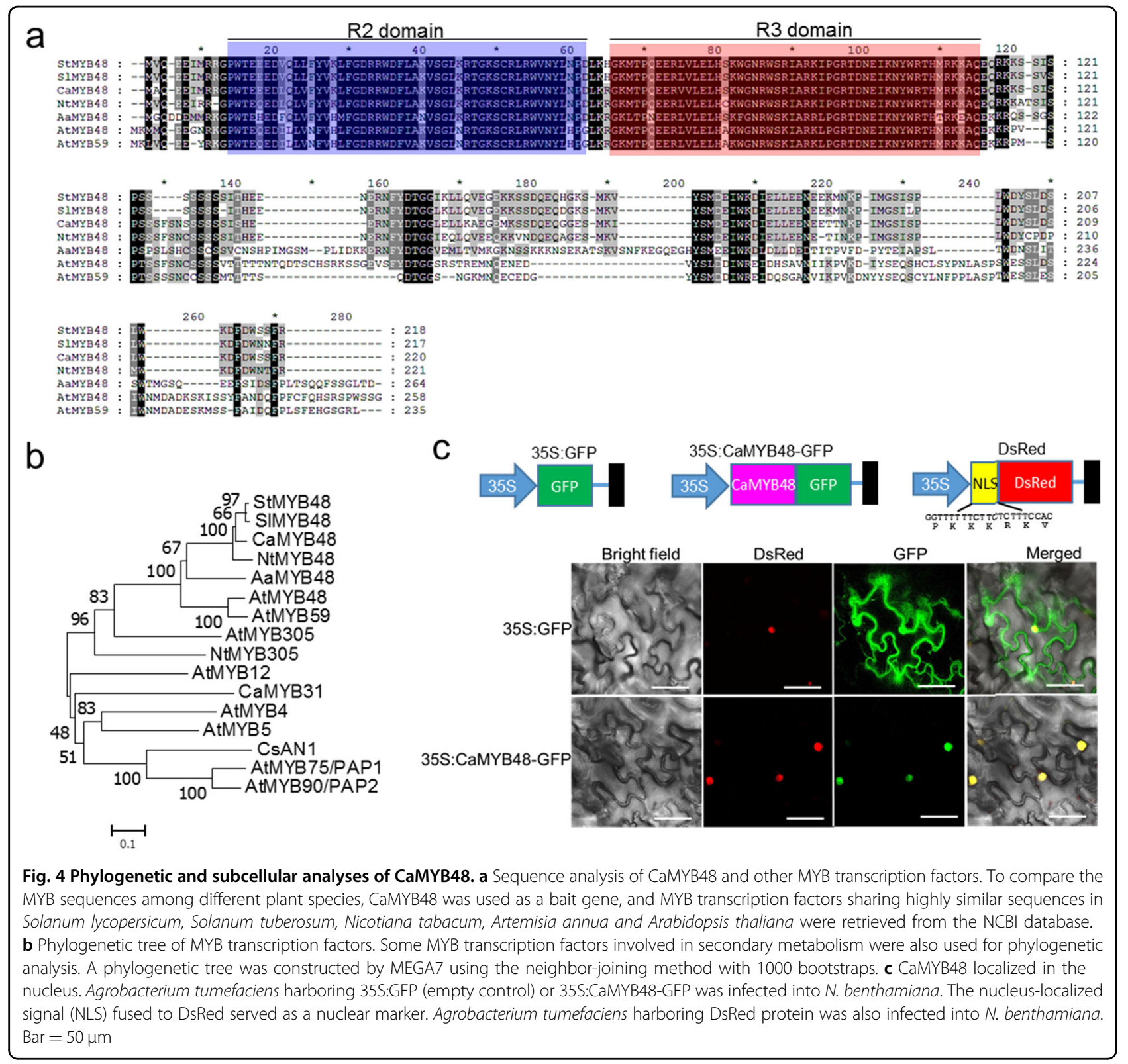

network genes identified in the MEblack module, strongly supporting that CaMYB48 regulates certain coexpressed CBGs to control capsaicinoid biosynthesis.

\section{CaMYB48 can directly bind to and activate the transcription of CBG promoters}

Since CaMYB48-coexpressed CBGs were downregulated in CaMYB48-silenced plants, we hypothesized that these genes may be directly targeted by CaMYB48. Two key CBGs, AT3 and KasIa, were selected to verify the hypothesis. To determine whether AT3a and KasIa are directly targeted by CaMYB48, we performed a yeast onehybrid $(\mathrm{Y} 1 \mathrm{H})$ analysis. The promoters of KasIa and AT3a were ligated into the pAbAi vector to generate bait vectors (pAbAi-ProKasIa and pAbAi-ProAT3a). CaMYB48 was ligated into pGADT7 to generate a prey vector. Subsequently, the bait and prey vectors were transformed into the $\mathrm{Y} 1 \mathrm{H}$ Gold yeast strain and screened on $\mathrm{SD} /$-Leu medium containing a given concentration of aureobasidin A. The results indicated that CaMYB48 associates with the KasIa and AT3a promoters in yeast. The results also indicated that the $\mathrm{Y} 1 \mathrm{H}$ Gold yeast strain cotransformed with the AD-CaMYB48 vector and that the promoters of KasIa and AT3a grew well on medium containing AbA (Fig. 7a). In contrast, the yeast cells cotransformed with empty vector, and the CBG promoters did not grow well on the AbA-containing medium. These results indicated that CaMYB48 is associated 


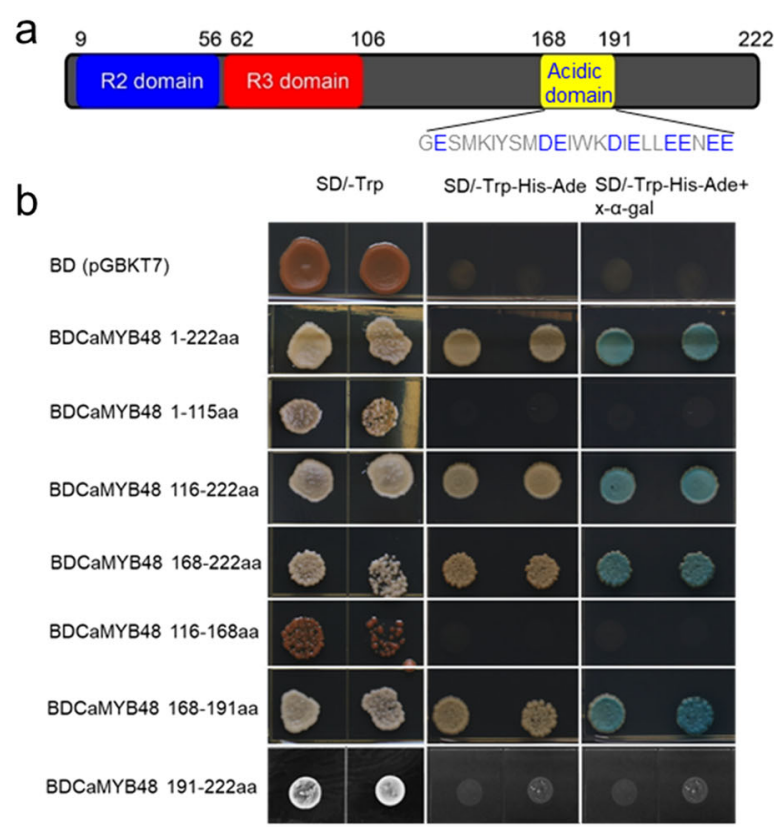

C

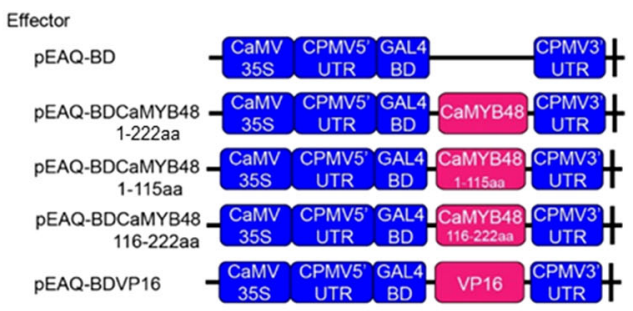

Reporter

GAL-LUC
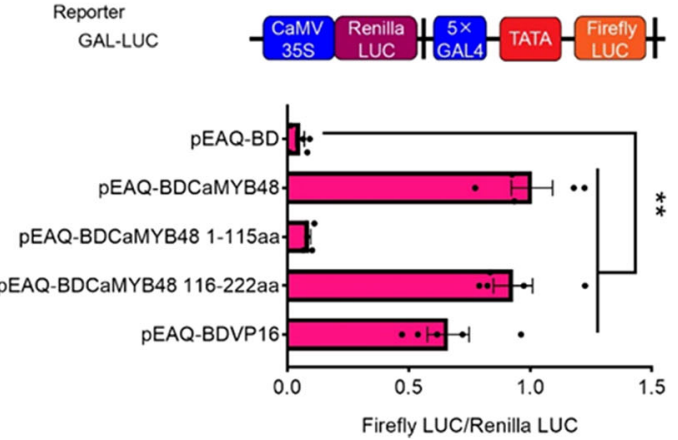

Fig. 5 CaMYB48 is a transcription activator. a Schematic diagram of the CaMYB48 domain. Within the acidic domain, the acidic amino acids are marked in blue. $\mathbf{b}$ Activation analysis of CaMYB48 in yeast. Full-length and truncated CaMYB48 was used for activation analysis. The number shown on the right indicates the protein region used for activation analysis. Auxotroph plates of SD/-Leu-His-Ade (middle) and SD/-Leu-His-Ade-x-a-gal (right) showing transcriptional activation of protein. c CaMYB48 process transcriptional activation in planta. The number shown on the right indicates the protein region used for analysis in N. benthamiana leaves. The transcriptional activator VP16 was used as the positive control. Data represent the mean \pm SD $(n=5)$. Student's $t$ test was used to identify significant differences compared to the empty vector control $(* * P<0.01)$

with the $A T 3 a$ and KasIa promoters in yeast. To confirm whether the transcription of KasIa and the AT3a promoter can be activated by CaMYB48 in vivo, a dual-LUC reporter assay was performed. The upstream start codons of $1843 \mathrm{bp}$ of AT3a and $1917 \mathrm{bp}$ of KasIa were used for assessment. Analysis of relative firefly LUC activity showed that the AT3a and KasIa promoter transcription levels were strongly activated by CaMYB48 in $N$. benthamiana leaves (Fig. 7b). R2R3 MYB transcription factors are known for binding to the MYB cis-element of target genes to execute transcriptional regulation processes $^{37}$. Therefore, we analyzed the AT3a promoter sequence and identified several MYB cis-elements. To further investigate whether CaMYB48 can directly bind to the MYB cis-elements located at -849 to $-841 \mathrm{bp}$ upstream of the $A T 3 a$ start codon, EMSAs were carried out. To determine whether CaMYB48 can bind to the AT3a promoter MYB cis-element, an electrophoretic mobility shift assay (EMSA) was carried out. The fulllength coding sequence of CaMYB48 was ligated into pMAL-c2X and transformed into BL21-competent E. coli. The recombinant maltose-binding protein (MBP) of CaMYB48 was expressed and purified with amylose resin (Fig. 7c). As the labeled probes were incubated with CaMYB48 recombinant proteins, the probes exhibited slowed bands, and the binding clearly decreased following incubation with unlabeled probes as competitors (Fig. 7d). Taken together, our results indicate that CaMYB48 targets the KasIa and $A T 3 a$ promoters by directly binding to their promoter-binding cis-elements.

\section{Discussion}

Coexpression facilitates the identification of capsaicinoidrelated modules

Plant biosynthesis of specialized metabolites is often limited to specific tissues or exclusively occurs in response to environmental stimuli ${ }^{38}$. Regulation of specialized metabolite biosynthesis seems to be controlled at the transcriptional level, which is generally dependent on the interaction of DNA-related mechanisms and the activity of transcription factors that may act in a combinatorial manner ${ }^{35,38}$. Capsaicinoids serve as defensive compounds to serve as deterrents against microorganisms and mammals to protect seeds from damage ${ }^{4,5,21}$. Pepper is a nonmodel horticultural crop, and the mining of biosynthetic genes and transcription regulators is still hindered ${ }^{4}$. Previously, the identification of the capsaicinoid biosynthesis-related genes $A T 3 a$, KasIa, CCoMTa, AMT, $K R$, and MYB31 was mainly based on genetic mapping, VIGS, and histochemistry approaches ${ }^{3,5}$. Omics-based 


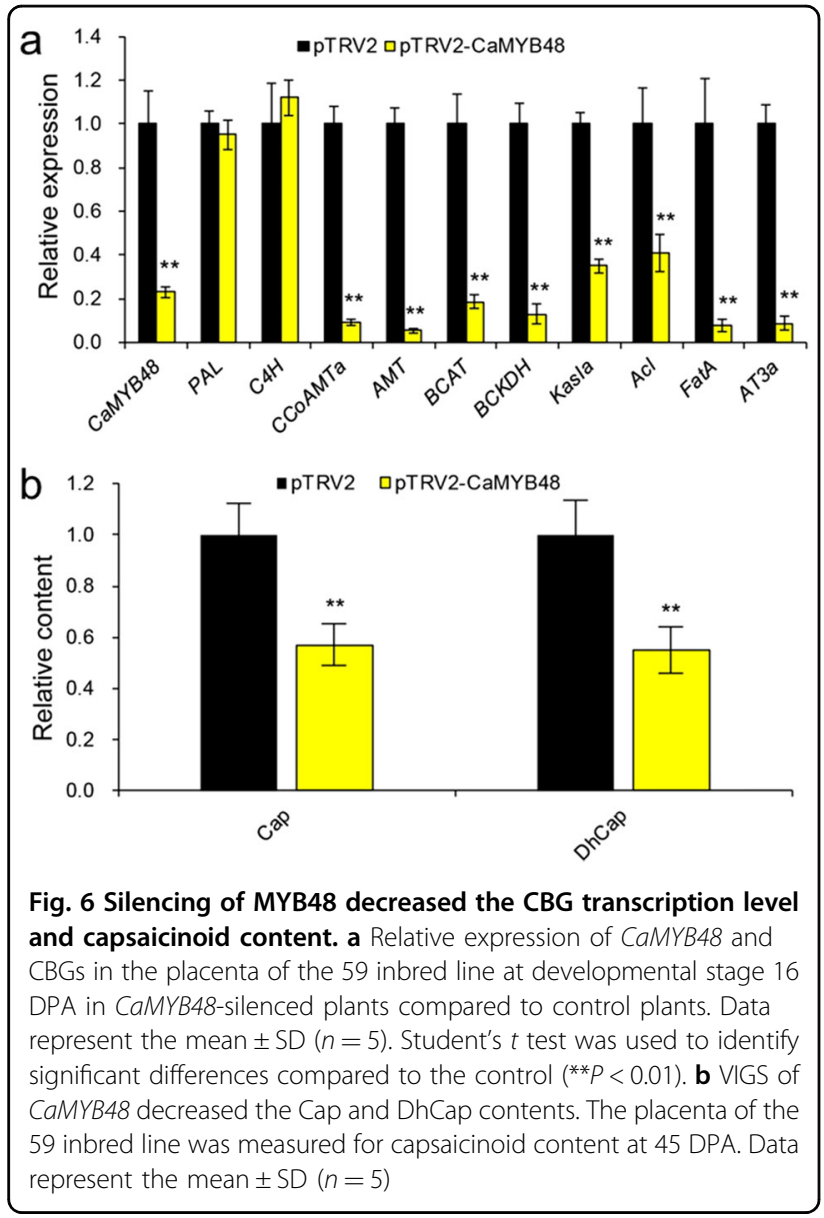

approaches have become available, facilitating research on nonmodel pepper plants. In this study, through WGCNA, we identified a MEblack module that exhibited a tight correlation of gene expression patterns with capsaicinoid biosynthesis (Fig. 1). Notably, a larger number of functionally characterized CBGs, such as AMT, AT3, KasIa, and $K R$, were identified in this module. Prior to this study, no coexpression network analysis-based strategy had been adopted to identify the CBGs and related transcriptional regulators. Compared with the biosynthesis pathways of other specialized metabolites, the capsaicinoid biosynthesis pathway is fairly complicated, since the production of capsaicinoids occurs via the condensation of precursors derived from phenylpropanoid and branched-chain fatty acid pathways. Based on bioinformatic and transcriptomic analyses, more than 50 genes were proposed to be involved in capsaicinoid biosynthesis. Via WGCNA, 14 CBGs were detected in the MEblack module, and most of them belonged to pathways downstream of biosynthetic genes. Presumably, the expression of most MEblack module CBGs exhibited tissue specificity and developmental stage regulation patterns (Fig. 1). Indeed, compared to the expression of MEblack module CBGs, the expression of some genes, such as $P A L, H C T$, and ACS, did not show apparent tissue specificity. We speculate that some of these coexpressed CBGs in the MEblack module encode key enzymes to determine the enzyme reaction rate. This property can be inferred from the results in which the capsaicinoid content was determined by the key CBGs, including $A M T$, KasIa, Acl, and $A T 3 a$, at the transcriptional level ${ }^{3,5}$. Coregulation has been observed for genes across multiple pathways of specialized metabolism, such as etoposide aglycone ${ }^{31}$, steroidal glycoalkaloids $^{39}$, and cucurbitacin ${ }^{40}$. These tight gene-tometabolite correlations were also reflective of predicted fluxes through the relevant pathways. To date, more than 22 capsaicinoids have been identified in hot pepper, but the genes responsible for the diversification of capsaicinoids have not been elucidated. The identified MEblack module with a tight gene-to-metabolite correlation will be a heavily studied topic in future research on the genes responsible for diversified capsaicinoids.

\section{CaMYB48 is a transcription factor involved in capsaicinoid biosynthesis}

Most MYB family proteins function as transcription factors with varying numbers of MYB repeat domains, conferring their ability to bind to DNA. The MYB family has selectively expanded in plant species, particularly through the large family of R2R3 MYBs. R2R3 MYB transcription factors have been documented to regulate different classes of specialized metabolites in plants, such as phenylpropanoids ${ }^{37}$, betaine ${ }^{41}$, glucosinolates ${ }^{42}$, and carotenoids ${ }^{43}$. These MYB factors appear to function in the coordinated control of metabolic genes, since they display similar expression patterns ${ }^{44}$. In this study, through WGCNA, we identified an MYB transcription factor, CaMYB48, that functions as a transcriptional activator to regulate capsaicinoid biosynthesis. The nonpungent pepper cultivar ECW has a 2.5-kb deletion in $A T 3 a$ that spans the region from the promoter to the first exon ${ }^{4}$. Expression analysis indicated that $A T 3 a$ was highly expressed in the pungent pepper cultivar CM334 and was barely expressed in the nonpungent pepper cultivar ECW. However, CaMYB48 was also highly expressed in cultivar ECW, and all other CBGs showed similar expression except for $A T 3 a$. These results indicate that the lack of pungency in ECW resulted from the loss of $A T 3 a$ expression without changes in the expression of CaMYB48 and other CBGs.

Although the sequence identity of CaMYB48 and its orthologs is highly conserved in Solanaceae plants, the functions of these orthologs in other Solanaceae plants have not been determined (Fig. 4). BLASTP analysis revealed that the MYB transcription factor from other plant species most closely related to CaMYB48 shared a fairly low sequence identity $(<50 \%$ amino acid sequence 


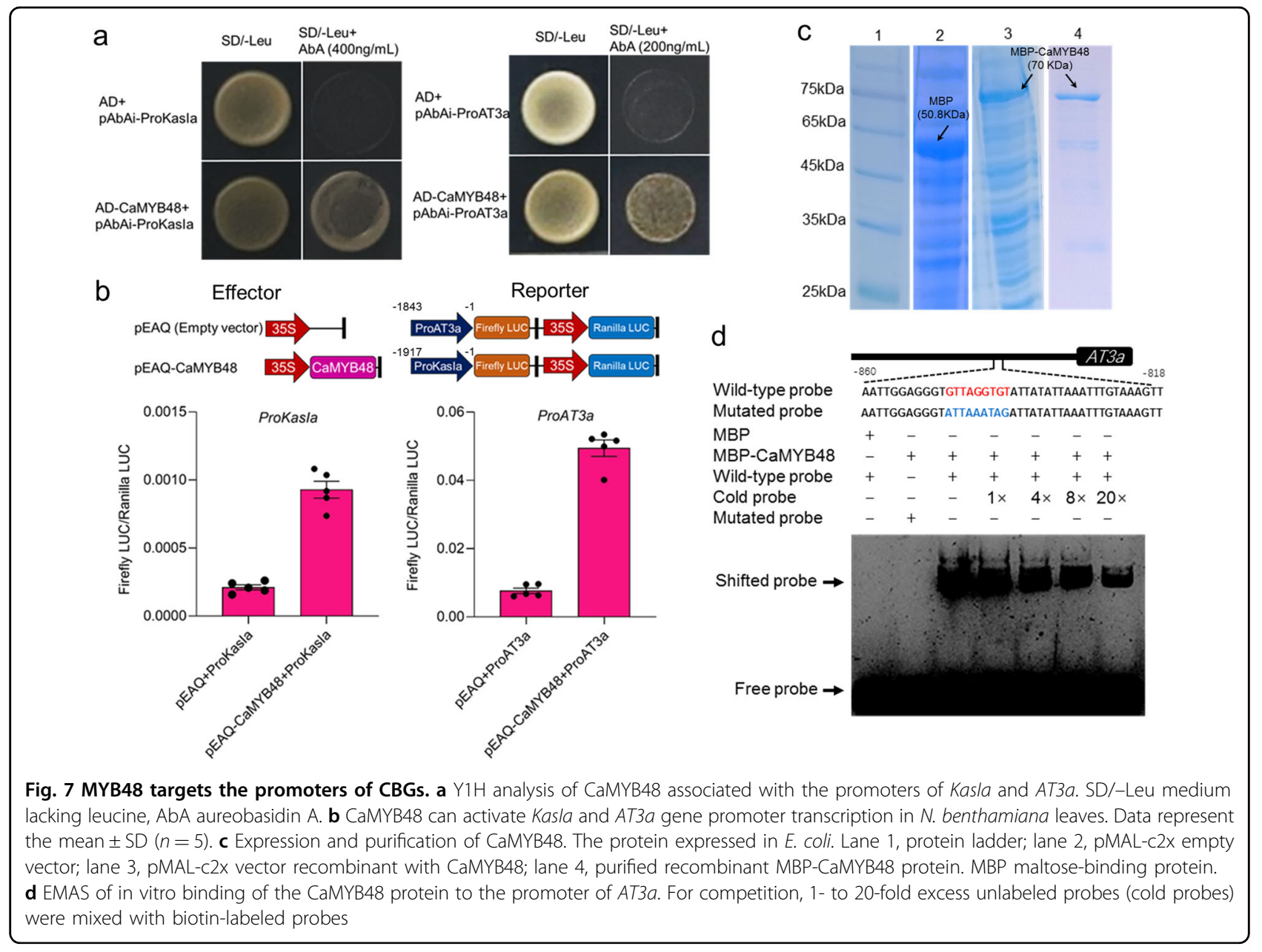

identity). In particular, the C-terminus of the amino acid sequence exhibited highly variable features among these proteins (Fig. 4a), which are recognized as crucial for defining MYBs from different subgroups ${ }^{37,45}$. For example, the Arabidopsis MYB transcription factor most closely related to CaMYB48 is AtMYB48, which exhibits only $53 \%$ sequence similarity. In addition, it is worth noting that the expression of CaMYB48 orthologs in different species displayed distinct patterns. In this context, Solanaceae MYB48 and the nearest MYB from other species appear to be derived from different ancestors. Alternatively, the functional and expression orthologs of CaMYB48 in different genera of plants underwent divergent evolution during or after speciation from ancestral species. However, the function of CaMYB48 orthologs in different species needs further study. We previously performed genetic analysis to identify MYB31 and functionally characterize its involvement in capsaicinoid biosynthesis through the regulation of structural gene biosynthesis ${ }^{5}$. Considering the complexity of capsaicinoid biosynthesis, the results of this study and prior results indicate that a variety of MYBs from different clades may be involved in capsaicinoid regulation in peppers. Whether additional cofactors interact with CaMYB48 to form regulatory complexes has not been determined.

In addition to the R2R3 DNA-binding domain, the Cterminus executes countless molecular functions that are absolutely crucial for biological function ${ }^{37}$. The Solanaceae-specific MYB transcription factor MYB31 has a Solanaceae-specific motif in the C-terminus (amino acids 213-234), and this motif performs its function as a transcriptional activation domain ${ }^{5}$. Unlike the highly conserved R2R3 DNA-binding domain, transcriptional activation domains seem to be intrinsically disordered, and low sequence conservation has made it challenging to identify the amino acid composition features that underlie their activity. In this study, characterization of the function of CaMYB48 as a transcriptional activator provides insight into this activation mechanism. Our results illustrated that the C-terminal at 168-191 aa, which is rich in acidic amino acids, is critical for the activation activity of CaMYB48. Deletion of this acidic amino acid activation 
motif completely abolished CaMYB48 activation activity both in yeast and in planta (Fig. 4). The acidic amino acid domains have been reported to be responsible for the ability of MYBs to execute transcriptional activation processes ${ }^{45}$. The Zea mays $\mathrm{C} 1$ protein was the first MYB transcription factor identified in plants and is characterized as a transcriptional activator by numerous acidic residues at the $\mathrm{C}$-terminus ${ }^{46,47}$. Recently, the Medicago truncatula MYB protein WP1 was elucidated to function as a transcriptional activator by directly regulating the expression of carotenoid biosynthetic genes through its Cterminal acidic activation motif ${ }^{43}$. These previous results revealed that the acidic region is essential for the activation activity of the MYB transcription factor. A BLASTP search and sequence analysis showed that the CaMYB48 acidic amino acid activation motif had no sequence similarity with any functionally characterized activation motifs. We speculate that convergent evolution of the MYB repeat domain ensures that the protein binds to the consensus DNA sequence, while divergent evolution of the activation motif enables the diversified functions of MYB transcription factors. However, the tested acidic amino acid activation motif contained 24 amino acid residues. We cannot rule out the possibility that shortening the tested amino acid residues would be sufficient for activation activity. Further shortening the amino acid residues and mutating the corresponding acidic amino acids enabled us to elucidate the activation mechanism.

In this study, through WGCNA, we identified a MEblack module related to capsaicinoid biosynthesis. The module provides candidate genes for the identification of new biosynthetic genes relevant to capsaicinoids. We elucidated that CaMYB48 functions as a transcriptional activator to regulate capsaicinoid biosynthesis. The identification of CaMYB48 provides a foundation for further research investigating the transcriptional regulatory networks for capsaicinoid biosynthesis in peppers and offers mechanistic insights into the evolution of plant secondary metabolism.

\section{Materials and methods \\ Plant materials}

The pepper (C. annuum) 59 inbred line is an elite line with high yield, high pungency, resistance to diverse pathogens and good heat, and drought tolerance. Thirtyfive-day-old seedlings were transplanted into $25-\mathrm{cm}$ plastic pots. The plants were grown in a greenhouse with a daily temperature of $25-27^{\circ} \mathrm{C}$, nighttime temperature of $18-20^{\circ} \mathrm{C}$, relative humidity of $65 \%, 16 / 8$-h light/dark cycle, and light intensity of 6500 Lux. After pepper fruit development to the selected stages, the fruits were sampled. The fruits were dissected to separate the pericarp, seeds, and placenta. The samples were frozen in liquid nitrogen and stored in a $-80^{\circ} \mathrm{C}$ freezer.

\section{Identification of coexpression modules and visualization of gene expression}

CM334 is a Mexican landrace that has consistently exhibited high levels of resistance to diverse pathogens. This landrace has been extensively used in hot pepper research and cultivar breeding, and its genome sequence was released in $2014^{4}$. The nonpungent cultivar ECW has a large deletion in $A T 3 a$ that spans the region from the promoter to the first exon, leading to loss of $A T 3 a$ (also known as $C S$ ) expression but without substantial changes in the expression of other genes in the biosynthetic pathway ${ }^{4}$. The 6421 inbred line was selected from a long-red-pepper landrace widely grown in Hunan Province, China. It is resistant to diverse pathogens and abiotic stress, and many F1 hybrids have been bred using 6421 as a female parent, which has been planted in many Chinese pepper-growing areas. The transcriptome data of pepper (C. annuum) cultivar CM334 (pungent), cultivar ECW (nonpungent) and inbred line 6421 (pungent) were retrieved from public databases ${ }^{4,36}$. These data include transcription data of genes from different tissues and fruits at different developmental stages. A gene coexpression network was built using the WGCNA package in $R^{33}$. The networks were visualized using Cytoscape v.3.6.0 $0^{48}$.

For high-throughput display of the expression levels of the assigned genes, heat maps were created. Related gene expression values were retrieved from public databases; the heat maps were plotted with the pheatmap package in $\mathrm{R}$.

\section{RNA extraction}

Quantitative real-time reverse transcription PCR (RTPCR) analysis of gene expression in different tissues was performed. Roots, leaves, stems, and apical buds were collected from 30-day-old seedlings. Fully blossomed flowers were collected from 80-day-old plants. Seeds and pericarp were collected from 16-DPA fruit, and the placenta was collected at different fruit developmental stages. Total RNA was extracted from the samples using a HiPure HP Plant RNA Mini Kit (Magen, China). The RNA samples were used for further analysis as described below.

\section{Determination of Cap and DhCap contents}

To determine the Cap and DhCap contents in the placenta of 59 inbred line pepper fruits from the indicated samples, the extraction and quantification of Cap and DhCap were performed as previously reported ${ }^{5}$. Briefly, $\sim 0.1$-g dry weight samples were extracted with $10 \mathrm{~mL}$ of a methanol/tetrahydrofuran (1:1) extract solution in a test tube at room temperature for $24 \mathrm{~h}$. The Cap and DhCap standards (Sigma-Aldrich) or extracts were injected into an XSelect HSS C-18 SB column $(4.6 \times 250 \mathrm{~mm}, 5 \mu \mathrm{m}$, Waters Technologies, USA) and separated using 20\% water (A) and $80 \%$ methanol (B) as mobile phases on a 
Waters Alliance Series HPLC system (Waters Technologies, USA). Detection was performed at $280 \mathrm{~nm}$ for Cap and DhCap.

\section{Sequence alignment and phylogenetic tree construction}

To retrieve the MYB transcription factor sequences from other plant species, CaMYB48 was used as the bait gene to blast against the NCBI, Sol Genomics Network and TAIR databases. The MYB transcription factor sequences were aligned by CLUSTALX 2 with the default parameters, and the phylogenetic tree was constructed by MEGA 7.0 using the neighbor-joining method with 1000 bootstrap replicates ${ }^{49}$. The MYB transcription factor motifs displayed in this study were predicted using the online version of MEME.

\section{Quantitative real-time RT-PCR analysis}

Total RNA was extracted from the tissues as described, and $1 \mu \mathrm{g}$ of total RNA from each sample was subjected to reverse transcription using HiScript III RT SuperMix for qPCR (+gDNA wiper) eraser (Vazyme Biotech, China). AceQ Universal SYBR qPCR Master Mix (Vazyme Biotech, China) was applied for quantitative real-time PCR analysis. Analysis was performed on a LightCycler 480 Real-Time PCR System according to the manufacturer's instructions (Roche, Switzerland), and the program was run as previously described ${ }^{50}$. The transcript level of the reference gene actin was used to quantify the relative transcript level of each target gene in each sample ${ }^{51}$. The values are means of three biological replicates. The primers used in this study are listed in Supplementary Table 3.

\section{Subcellular localization}

The full-length coding sequence of CaMYB48 was cloned into the pEAQ-GFP vector and fused with GFP under the control of the CaMV $35 \mathrm{~S}$ promoter. The Agrobacterium tumefaciens strain GV3101 containing the corresponding constructs was infiltrated into young $N$. benthamiana leaves. After $N$. benthamiana leaves were inoculated with Agrobacterium tumefaciens for 3 days, the GFP signal was detected by confocal fluorescence microscopy (Carl Zeiss, Germany). The SV40 antigen (PKKKRKV) was used as a nuclear localization signal (NLS). To indicate the nuclei, the nucleotide sequence encoding the NLS was in frame with DsRed for nuclear targeting of the DsRed protein. The solution was infiltrated into $N$. benthamiana leaves. The primers used in this study are listed in Supplementary Table 3.

\section{Transcriptional activation analysis}

A yeast transcriptional activation assay was performed as described in the manufacturer's instructions for the Matchmaker Gold yeast two-hybrid system (Clontech,
USA). Full-length CaMYB48 or truncated CaMYB48 was cloned into pGBKT7 to generate BDCaMYB48. The BDCaMYB48 or the BD empty vector was then transformed into the Saccharomyces cerevisiae strain $\mathrm{Y} 2 \mathrm{H}$ Gold and incubated on SD/-Trp medium at $30^{\circ} \mathrm{C}$ for 3 days. The positive clones were picked and diluted in $0.9 \% \mathrm{NaCl}$ solution, and $10 \mu \mathrm{L}$ of each dilution was inoculated onto $\mathrm{SD} /$-Trp-His-Ade medium. After $3-5$ days, the clones were stained with $x-\alpha-G a l$ (Clontech, USA). The primers used in this study are listed in Supplementary Table 3.

The transcriptional activation analysis of CaMYB48 in planta was carried out according to a previous study ${ }^{5}$. Full-length or truncated coding sequences of CaMYB48 were cloned into the pEAQ-BD vector and fused with the GAL4 DNA-binding domain under the control of the CaMV $35 S$ promoter. The GAL-LUC reporter contained a firefly LUC gene driven by the minimal CaMV $35 \mathrm{~S}$ promoter and five GAL4-binding elements. The Renilla LUC gene under the control of CaMV $35 \mathrm{~S}$ on the same plasmid served as an internal control. The Agrobacterium tumefaciens strain GV3101 containing the effector or pEAQ$\mathrm{BD}$ and reporter was coinfiltrated into young $N$. benthamiana leaves. Three days after infection, the firefly LUC and Renilla LUC activities were measured with a dual-LUC reporter assay system (Promega, USA) on a Varioskan ${ }^{\mathrm{TM}}$ LUX multimode microplate reader (Thermo Fisher Scientific, USA). The primers used are listed in Supplementary Table 3.

\section{$\mathrm{Y} 1 \mathrm{H}$ assay}

The full-length coding sequence CaMYB48 was cloned into pGADT7 to generate prey AD-CaMYB48. The promoter fragments of KasIa and AT3a were ligated into the pAbAi vector to generate baits. The $\mathrm{Y} 1 \mathrm{H}$ experiment was carried out according to the manufacturer's protocol for the Matchmaker Gold Y1H library screening system (Clontech, USA). The primers used in this study are listed in Supplementary Table 3.

\section{VIGS analysis}

CaMYB48 coding sequence fragments that shared low similarity with other genes were cloned into PTRV2 to generate the silencing vector pTRV2-CaMYB48. The constructs included pTRV1, pTRV2, and pTRV2CaMYB48 of Agrobacterium tumefaciens strain GV3101. Agrobacterium tumefaciens containing pTRV2-CaMYB48 or pTRV2 and pTVR1 vectors was coinfiltrated into 35day-old seedlings of the 59 inbred line of pepper. The plants were grown in a greenhouse with a daily temperature of $24^{\circ} \mathrm{C}$, nighttime temperature of $18{ }^{\circ} \mathrm{C}$, relative humidity of $70 \%, 16 / 8$-h light/dark cycle, and light intensity of 6500 Lux. Total RNA was extracted from 16DPA fruit placentas for expression analysis to evaluate 
silencing efficiency. The 45 PDA fruit placentas were used for Cap and DhCap content measurements. The primers used in this study are listed in Supplementary Table 3.

\section{Dual-LUC assay}

The upstream start codons of $A T 3 a(1843 \mathrm{bp})$ and KasIa (1917 bp) were PCR amplified from genomic DNA of the pepper inbred line 59 and cloned into pGreenII0800 to serve as reporters. Within this vector, the Renilla gene under the control of a $35 \mathrm{~S}$ promoter was used for the normalization of transfection efficiency. Full-length CaMYB48 was PCR amplified from cDNA from the placenta of pepper inbred line 59 and cloned into the pEAQ vector under the CaMV $35 \mathrm{~S}$ promoter to serve as an effector. The Agrobacterium tumefaciens strain GV3101 containing the CaMYB48 effector and the corresponding reporters were coinfiltrated into young $N$. benthamiana leaves. After incubation for 3 days, the firefly LUC and Renilla LUC activities were measured with a dual-LUC reporter assay system (Promega, USA) on a Varioskan ${ }^{\mathrm{TM}}$ LUX (Thermo Fisher Scientific, USA). Activity is expressed as the ratio of firefly LUC activity to Renilla LUC activity. The primers used in this study are listed in Supplementary Table 3.

\section{Electrophoretic mobility shift assays (EMSAs)}

To express recombinant protein, full-length CaMYB48 was cloned into the pMAL-c2-x vector and fused in frame with the MBP tag. The constructs transformed into E. coli strain BL21 were grown in liquid medium to an OD at $600 \mathrm{~nm}$ of 0.4 , treated with 1-mM IPTG to induce expression, and grown for $16 \mathrm{~h}$ at $16^{\circ} \mathrm{C}$ with $130 \mathrm{rpm}$. The recombinant protein was purified with amylose beads according to the manufacturer's instructions (NEB, USA). The probe DNA containing the wild-type cis-element and mutated cis-element probes was labeled using a Pierce Biotin 3' End DNA Labeling Kit (Thermo Fisher Scientific, USA). EMSA was performed using a LightShift Chemiluminescent EMSA Kit (Thermo Fisher Scientific, USA) according to the manufacturer's instructions. The primers used in this study are listed in Supplementary Table 3.

\section{Acknowledgements}

This work was supported by the National Key Research and Development Program (2018YFD1000800) and the National Natural Science Foundation of China (31572124).

\section{Author details}

'Key Laboratory of Biology and Genetic Improvement of Horticultural Crops (South China), Ministry of Agriculture and Rural Affairs, College of Horticulture, South China Agricultural University, Guangzhou 510642, China. Jiangxi Agricultural Engineering College, Zhangshu 331200 Jiangxi, China. ${ }^{3}$ Guangdong Helinong Seeds, Co., Ltd., Shantou 515800 Guangdong, China. ${ }^{4}$ Guangdong Helinong Agricultural Research Institute, Co., Ltd., Shantou 515800 Guangdong, China. ${ }^{5}$ Henry School of Agricultural Science and Engineering, Shaoguan University, Guangdong 512005, China. ${ }^{6}$ Peking
University - Southern University of Science and Technology Joint Institute of Plant and Food Sciences, Department of Biology, Southern University of Science and Technology, Shenzhen 518055, China

\section{Conflict of interest}

The authors declare that they have no conflict of interest.

Supplementary Information accompanies this paper at (https://doi.org/ 10.1038/s41438-020-00381-2).

Received: 6 March 2020 Revised: 18 June 2020 Accepted: 13 July 2020 Published online: 01 October 2020

\section{References}

1. Bosland, P. W. \& Votava, E. J. (eds) Peppers: Vegetable and Spice Capsicums (CABI, 2012).

2. Caterina M. J. et al. The capsaicin receptor: a heat-activated ion channel in the pain pathway. Nature 389, 816-824 (1997).

3. Aluru M. R. et al. Differential expression of fatty acid synthase genes, Acl, Fat and Kas, in capsicum fruit. J. Exp. Bot. 54, 1655-1664 (2003).

4. Kim S. et al. Genome sequence of the hot pepper provides insights into the evolution of pungency in capsicum species. Nat. Genet. 46, 270-276 (2014).

5. Zhu Z. et al. Natural variations in the MYB transcription factor MYB31 determine the evolution of extremely pungent peppers. N. Phytol. 223, 922-938 (2019).

6. Bennett, D. \& Kirby, G. Constitution and biosynthesis of capsaicin. J. Chem. Soc. C: Org. 1968, 442-446 (1968).

7. Iwai, K, Suzuki, T. \& Fujiwake, H. Formation and accumulation of pungent principle of hot pepper fruits, capsaicin and its analogues, in Capsicum annuun var. annuun Cv. karayatsubusa at different growth stages after flowering. Agric. Biol. Chem. 43, 2493-2498 (1979).

8. Stewart $C$. Jr. et al. The Pun 1 gene for pungency in pepper encodes a putative acyltransferase. Plant J. 42, 675-688 (2005).

9. Koeda S. et al. Mutation in the putative ketoacyl-ACP reductase CaKR1 induces loss of pungency in Capsicum. Theor. Appl. Genet. 132, 65-80 (2019).

10. Liu, S., Li, W., Wu, Y., Chen, C. \& Lei, J. De novo transcriptome assembly in chili pepper (Capsicum frutescens) to identify genes involved in the biosynthesis of capsaicinoids. PLoS ONE 8, e48156 (2013).

11. Liu S. et al. RNA-sequencing tag profiling of the placenta and pericarp of pungent pepper provides robust candidates contributing to capsaicinoid biosynthesis. Plant Cell Tiss. Org. 110, 111-121 (2012).

12. Abraham-Juárez, M. R. Rocha-Granados, M. C., López, M. G., Rivera-Bustamante, R. F. \& Ochoa-Alejo, N. Virus-induced silencing of Comt, pAmt and Kas genes results in a reduction of capsaicinoid accumulation in chili pepper fruits. Planta 227, 681-695 (2008).

13. Ogawa K. et al. Evidence of capsaicin synthase activity of the Pun1-encoded protein and its role as a determinant of capsaicinoid accumulation in pepper. BMC Plant Bio. 15, 93 (2015).

14. Tewksbury J. J. et al. Evolutionary ecology of pungency in wild chilies. Proc Natl Acad. Sci. U. S. A. 105, 11808-11811 (2008).

15. Lejeune, M. P. G. M., Kovacs, E. M. R. \& Westerterp-Plantenga, M. S. Effect of capsaicin on substrate oxidation and weight maintenance after modest bodyweight loss in human subjects. Brit. J. Nutr. 90, 651-659 (2007).

16. Ludy, M. J., Moore, G. E. \& Mattes, R. D. The effects of capsaicin and capsiate on energy balance: critical review and meta-analyses of studies in humans. Chem. Senses 37, 103-121 (2012).

17. Wee, L. T., Muhamad, I. I., Abang, Z. D. N. \& Khairuddin, N. Evaluation of capsaicinoids extracts as bioactive substance for antimicrobial films. Jurnal Teknologi 64, 69-74 (2013).

18. Busker, R. W. \& Van Helden, H. P. M. Toxicologic evaluation of pepper spray as a possible weapon for the Dutch police force: risk assessment and efficacy. Am. J. Foren. Med. Path. 19, 309-316 (1998).

19. Sun B. et al. Jasmonate-Inducible R2R3-MYB transcription factor regulates capsaicinoid biosynthesis and stamen development in Capsicum. J. Agr. Food Chem. 67, 10891-10903 (2019).

20. Garruña-Hernández, R.r Monforte-González, M., Canto-Aguilar, A., VázquezFlota, F. \& Orellana, R. Enrichment of carbon dioxide in the atmosphere increases the capsaicinoids content in Habanero peppers (Capsicum chinense Jacq.). J. Agr. Food Chem. 93, 1385-1388 (2013). 
21. Qin C. et al. Whole-genome sequencing of cultivated and wild peppers provides insights into Capsicum domestication and specialization. Proc. Natl Acad. Sci. U. S. A. 111, 5135-5140 (2014).

22. Yu Z. X. et al. The Jasmonate-responsive AP2/ERF transcription factors AaERF1 and AaERF2 positively regulate artemisinin biosynthesis in Artemisia annua $\mathrm{L}$. Mol. Plant 5, 353-365 (2012)

23. Ogawa S. et al. OsMYC2, an essential factor for JA-inductive sakuranetin production in rice, interacts with MYC2-like proteins that enhance its transactivation ability. Sci. Rep. 7, 40175 (2017)

24. Fits, L. \& Memelink, J. ORCA3, a jasmonate-responsive transcriptional regulator of plant primary and secondary metabolism. Science 289, 295-297 (2000).

25. Butelli E. et al. Enrichment of tomato fruit with health-promoting anthocyanins by expression of select transcription factors. Nat. Biotechnol. 26, 1301-1308 (2008).

26. Sun B. et al. Purple foliage coloration in tea (Camellia sinensis L.) arises from activation of the R2R3-MYB transcription factor CsAN1. Sci. Rep. 6, 32534 (2016).

27. Butelli E. et al. Retrotransposons control fruit-Specific, cold-dependent accumulation of anthocyanins in blood oranges. Plant Cell 24, 1242-1255 (2012).

28. Arce-Rodríguez, M. L. \& Ochoa-Alejo, N. An R2R3-MYB transcription factor regulates capsaicinoid biosynthesis. Plant Physiol. 174, 1359-1370 (2017).

29. Ruan, J., Dean, A. K. \& Zhang, W. A general co-expression network-based approach to gene expression analysis: comparison and applications. BMC Syst. Biol. 4, 8-8 (2010).

30. Higashi, Y. \& Saito, K. Network analysis for gene discovery in plant-specialized metabolism. Plant Cell Environ. 36, 1597-1606 (2013).

31. Lau, W. \& Sattely, E. S. Six enzymes from mayapple that complete the biosynthetic pathway to the etoposide aglycone. Science 349, 1224-1228 (2015).

32. Cárdenas P. D. et al. GAME9 regulates the biosynthesis of steroidal alkaloids and upstream isoprenoids in the plant mevalonate pathway. Nat. Commun. 7 10654-10654 (2016)

33. Langfelder, P. \& Horvath, S. WGCNA: an R package for weighted correlation network analysis. BMC Bioinform. 9, 559-559 (2008).

34. Shahan R. et al. Consensus coexpression network analysis identifies key regulators of flower and fruit development in wild strawberry. Plant Physiol. 178, 202-216 (2018).

35. Allan, A. C., Hellens, R. P. \& Laing, W. A. MYB transcription factors that colour our fruit. Trends Plant Sci. 13, 99-102 (2008).

36. Liu F. et al. PepperHub, an informatics hub for the chili pepper research community. Mol. Plant 10, 1129-1132 (2017).
37. Dubos C. et al. MYB transcription factors in Arabidopsis. Trends Plant Sci. $\mathbf{1 5}$ 573-581 (2010)

38. Colinas, M. \& Goossens, A. Combinatorial transcriptional control of plant specialized metabolism. Trends Plant Sci. 23, 324-336 (2018).

39. Itkin M. et al. Biosynthesis of antinutritional alkaloids in solanaceous crops is mediated by clustered genes. Science 341, 175-179 (2013).

40. Shang $Y$. et al. Biosynthesis, regulation, and domestication of bitterness in cucumber. Science 346, 1084-1088 (2014).

41. Hatlestad G. J. et al. The beet Y locus encodes an anthocyanin MYB-like protein that activates the betalain red pigment pathway. Nat. Genet. 47, 92-96 (2015).

42. Yi, L., Chen, H. C., Cao, B. H. \& Lei, J. J. Molecular characterization of MYB28 involved in aliphatic glucosinolate biosynthesis in Chinese kale (Brassica oleracea var. alboglabra Bailey). Front. Plant Sci. 8, 1083 (2017).

43. Gigolashvili, T., Engqvist, M., Yatusevich, R., Muller, C. \& Flugge, U. I. HAG2/ MYB76 and HAG3/MYB29 exert a specific and coordinated control on the regulation of aliphatic glucosinolate biosynthesis in Arabidopsis thaliana. $N$. Phytol. 177, 627-642 (2008).

44. Meng Y. et al. The MYB activator WHITE PETAL1 associates with MtTT8 and MtWD40-1 to regulate carotenoid-derived flower pigmentation in Medicago truncatula. Plant Cell 31, 2751-2767 (2019).

45. Soler M. et al. The Eucalyptus grandis R2R3-MYB transcription factor family: evidence for woody growth-related evolution and function. N. Phytol. 206, 1364-1377 (2015)

46. Millard, P. S., Kragelund, B. B. \& Burow, M. R2R3 MYB transcription factorsfunctions outside the DNA-binding domain. Trends Plant Sci. 24, 934-946 (2019).

47. Paz-Ares, J., Ghosal, D., Wienand, U., Peterson, P. A. \& Saedler, H. The regulatory c1 locus of Zea mays encodes a protein with homology to myb protooncogene products and with structural similarities to transcriptional activators. EMBO J. 6, 3553-3558 (1987).

48. Shannon P. et al. Cytoscape: a software environment for integrated models of biomolecular interaction networks. Genome Res. 13, 2498-2504 (2003).

49. Kumar, S., Stecher, G. \& Tamura, K. MEGA7: molecular evolutionary genetics analysis version 7.0 for bigger datasets. Mol. Biol. Evol. 33, 1870-1874 (2016).

50. Zhu Z. S. et al. Pyramiding of AtEDT1/HDG11 and Cry2Aa2 into pepper (Capsicum annuum L.) enhances drought tolerance and insect resistance without yield decrease. Plant Cell Tiss. Org. 120, 919-932 (2015).

51. Chen C. et al. CaMF2, an anther-specific lipid transfer protein (LTP) gene, affects pollen development in Capsicum annuum L. Plant Sci. 181, 439-448 (2011). 